\title{
FACTORES PREDICTORES \\ DE LA PARTICIPACIÓN \\ EN UN PROGRAMA DE «SCREENING» \\ DE CÁNCER DE MAMA: \\ IMPLICACIÓN DEL MODELO \\ DE CREENCIAS DE SALUD Y DE LAS \\ VARIABLES SOCIODEMOGRÁFICAS
}

\section{Lourdes Lostao}

Universidad Pública de Navarra

E-mail:1lostao@unavarra.es

\section{RESUMEN}

El presente artículo tiene por objeto analizar la posible implicación de las variables del modelo de creencias de salud y las variables sociodemográficas en la participación en un programa de «screening» de cáncer de mama, el cual se ha llevado a cabo en Navarra, dentro de la Red Piloto Europea de Programas de Detección Precoz de Cáncer de Mama, dirigido a mujeres de 45-65 años de edad. Se utilizó una muestra total de 708 mujeres normales, de las cuales 512 tenían la condición de participantes en el Programa de Detección Precoz del Cáncer de Mama en Navarra, en tanto que las restantes 196 no acudieron al mismo. A todas ellas se les administró un cuestionario de forma individual.

De acuerdo con el modelo de creencias de salud, las variables analizadas fueron: Severidad, Susceptibilidad y Beneficios. Además, se estudiaron las variables sociodemográficas siguientes: edad, estado civil, nivel asociativo y status social. Tras la realización de análisis de regresión logística se constató que las variables severidad, estado civil, nivel asociativo y status social están asociadas a la participación en el programa de «screening» de cáncer de mama.

\section{INTRODUCCIÓN}

Desde comienzos de la Sociología de la Medicina y posteriores estudios en los EE.UU. (Rosenblatt y Suchman, 1964; Dutton, 1978, 1979, 1986; Cockerham et al., 1988) y en Alemania (Siegrist y Bertram, 1970, 1971) muchos trabajos se han centrado en la investigación de las diferencias en la utilización de servicios de salud preventiva y en los factores que intervienen a la 
hora de llevar a cabo conductas de salud preventiva por parte de grupos de población. En este sentido, la salud preventiva se ha referido a exámenes físicos rutinarios, salud prenatal, chequeos dentales, «screening» para enfermedades cardiovasculares y cáncer, así como otros servicios dirigidos a obtener buena salud y a prevenir la enfermedad mediante detección precoz, o bien a minimizar los efectos de la enfermedad si ésta ocurre.

En la actualidad, el cáncer de mama es una de las primeras causas de muerte de la población femenina en los países industrializados. En Estados Unidos, por ejemplo, una de cada nueve mujeres desarrollará cáncer de mama a lo largo de su vida (American Cancer Society, 1996; Urban et al., 1994; Kessler et al., 1991). En España, el cáncer de mama representa globalmente un 18 por 100 de todas las muertes por cáncer de las mujeres españolas (Indicadores de salud, 1993). La influencia que la mortalidad por cáncer de mama tiene en la mortalidad global femenina en España (Ascunce, 1991) y en otros países occidentales (De Waard, 1993; Coleman et al., 1993; García Arcal et al., 1994) ha impulsado el desarrollo de programas de detección precoz poblacionales para dicha enfermedad. Distintos estudios han demostrado la efectividad de tales programas de prevención secundaria («screening») mediante mamografía para reducir la mortalidad por cáncer de mama (Shapiro et al., 1971; Fletcher et al., 1993; Morrison, 1993; White et al., 1993). El método más efectivo para la detección precoz del cáncer de mama es la utilización del «screening» mediante mamografía de forma periódica, y su implementación a larga escala en unos grupos de edad específicos (Smith, 1992; Romans, 1993; White et al., 1993; Blamey et al., 1994; Ascunce et al., 1993; Bonfill et al., 1992; Borrás et al., 1994; Rodríguez et al., 1991; Luengo et al., 1997). En el cáncer de mama, el establecer un diagnóstico precoz de la enfermedad es clave, ya que este diagnóstico está directamente relacionado con los índices de supervivencia y con la calidad de vida de las mujeres afectadas.

Por lo que respecta a la edad en la cual una mujer debe comenzar a realizar chequeos mamográficos, y que éstos sean efectivos en cuanto a la reducción de mortalidad, no hay evidencia concluyente. Algunos autores recomiendan la mamografía para todas las mujeres de más de cuarenta años y que ésta se realice con una periodicidad de cada dos años (White et al., 1993). Otros autores, en cambio, señalan que la mamografía no reduce significativamente la mortalidad en mujeres menores de cincuenta años (Kerlikowske et al., 1995) o mayores de setenta años (Blamey et al., 1994). Algunos señalan que las mamografías son efectivas después de los cincuenta años y con una periodicidad anual (Dodd, 1992). La mayoría de los autores, no obstante, recomiendan que la mamografía debería repetirse con un intervalo de dos años (Blamey et al., 1994).

Sobre la base del aumento de la evidencia contrastada a favor del «screening» mamográfico del cáncer de mama, países como Gran Bretaña, Noruega y Suecia implantaron a nivel nacional programas de «screening» para el cáncer de mama. La detección precoz del cáncer de mama, centrado en la técnica de la 
mamografía, es una prioridad importante en los sistemas de salud de Europa, ya que resulta eficaz para detectar y combatir la enfermedad. También otro conjunto de programas locales han sido implementados en distintas regiones europeas, apoyados por la Comunidad Europea. En concreto, dentro del Programa Europa contra el Cáncer, se ha desarrollado y financiado varios proyectos piloto en Bélgica (Bruselas y Flandes), Francia (Estrasburgo), Grecia (Atenas), Irlanda (Dublín), España (Navarra), Italia (Florencia) y Portugal (Coimbra). (Programa Europa contra el Cáncer, CEE, 1986.) Dichos proyectos europeos están coordinados entre sí, intercambiando información y resultados. Tales programas, aunque son similares, tienen en cuenta las posibles divergencias relativas a características culturales y locales de sus propios servicios de salud, así como de sus ámbitos y culturas (Pas y Robra, 1993).

Dada la importancia de los programas preventivos, un factor relevante y primordial es la alta participación de las mujeres en dichos programas. Para conseguir una reducción mínimamente sustancial de la mortalidad, todo programa de detección precoz debe alcanzar una tasa elevada de participación de la población (Boyle et al., 1995; Ascunce et al., 1993; Donovan et al., 1988). Por eso, para conseguir el objetivo general de estos programas, es decir, reducir la mortalidad por cáncer de mama, es necesario obtener un alto nivel de participación de la población (Ascunce et al.,1994).

Estudios efectuados en España (Lostao, 1998; Lostao et al., 1996; 2001; Ascunce et al., 1994; Rodríguez et al., 1995; Luengo et al., 1996; Segura et al., 1998) y en otros países (Van den Heuvel, 1977; Vermost, 1978; Fink et al., 1972, 1968; French et al., 1982; Vernon et al., 1990; Calnan, 1984; Donato et al., 1991; Eardley y Elkind, 1990) han descrito diversas variables relacionadas con la participación en programas de «screening», así como diferencias sociales en los comportamientos de prevención. Se ha señalado que la participación es mayor en los grupos de edad más jóvenes, con superior nivel educativo, en las clases sociales superiores, y entre las mujeres casadas (Vernon et al., 1990; Lostao et al., 1996; Link et al., 1998). Donde ha sido posible apreciar diferencias significativas ha sido en las variables edad, estado civil, nivel asociativo y status social. También las creencias favorables hacia las actividades preventivas parecen constituir una serie de variables que favorece la participación (Vernon et al., 1990; Calnan, 1984; Taplin et al., 1989).

Como hemos señalado anteriormente, un importante modelo en la prevención de enfermedad y en la decisión de adoptar conductas de salud preventivas es el sistema de creencias de salud. Investigaciones realizadas muestran el poder del modelo de creencias de salud (Health Belief Model; en adelante, HBM) para explicar y predecir la utilización de servicios de salud preventivos (Rosenstock, 1966; Becker, 1974; McKinlay, 1972; Becker y Maiman, 1975; Becker y Rosenstock, 1989; Rosenstock, 1974; Becker, 1977, 1979; Janz y Becker, 1984; Reese y Smith, 1997).

Este modelo fue desarrollado inicialmente en los años cincuenta para explicar por qué las personas no llegaban a participar en programas de preven- 
ción o detección de enfermedades (Hochbaum, 1958; Rosenstock, 1966). Más adelante se amplió para explicar las respuestas de las personas a los síntomas (Kirscht,1974), así como la conducta de respuesta a las enfermedades diagnosticadas (Becker,1974). Investigaciones hechas en los años noventa siguen estudiando la calidad predictiva de las creencias de salud (Strecher, Champion y Rosenstock,1997). El modelo ha sido empleado con éxito en importantes investigaciones de conductas de salud preventiva, tales como la prevención dental (Gochman, 1971) y el tratamiento de la obesidad infantil (Becker et al., 1977) y del tabaquismo (Knight and Hay, 1989), así como las diferencias étnicas en el control de la hipertensión (Brown and Segal, 1996).

Un área de investigación relevante en la que se muestra una fuerte evidencia de asociación entre las creencias de salud y la participación en programas preventivos es la relacionada con la detección precoz del cáncer de mama. En concreto, las variables dentro del modelo de creencias de salud (Health Belief Model, HBM) han sido utilizadas en distintas investigaciones sociológicas sobre la participación en programas de detección precoz del cáncer de mama mediante mamografía (Calnan, 1984; Vernon et al., 1990; Thomas et al., 1996). Las tres variables más relevantes de este modelo son: (1) Susceptibilidad, que se refiere a la creencia de las personas sobre la posibilidad de contraer una enfermedad. (2) Severidad, que refleja la percepción de amenaza para la propia persona e incluye las evaluaciones de las posibles consecuencias médicas (muerte o discapacidad) y sociales (efectos de la enfermedad sobre el trabajo, la vida familiar y las relaciones sociales). (3) Beneficios, que hace referencia a la creencia sobre la efectividad de realizar conductas específicas para reducir la amenaza de la condición. El núcleo teórico del modelo de creencias de salud establece que una persona llevará a cabo un comportamiento de salud (de prevención, de participación, etc.) cuando se vea a sí misma como potencialmente vulnerable y vea la condición (la enfermedad en cuestión) como amenazante, esté convencida de la eficacia de la intervención y vea pocas dificultades en la puesta en práctica de la conducta de salud preventiva (Rosenstock, 1974).

Mediante la presente investigación pretendemos estudiar la relación entre estas variables de creencias de salud y las variables sociodemográficas con respecto a la participación en el programa de «screening» del Gobierno de Navarra. Una forma de estudiar tal relación consiste en analizar los índices de posibilidad de participación odds ratio (OR) asociados a dichas variables. Así, una de las finalidades de este estudio consiste en analizar los OR relacionados con ciertas variables sociodemográficas junto con las variables que han venido siendo propuestas como relevantes dentro del modelo de creencias de salud. 


\section{METODOLOGÍA}

\subsection{Caracteristicas de la muestra}

Se entrevistaron 708 mujeres normales; de ellas, 512 eran "participantes» en el Programa de Detección Precoz del Cáncer de Mama en Navarra, mientras que las 196 restantes eran «no participantes». Todas ellas habían sido incluidas dentro del Programa de Detección Precoz del Cáncer de Mama del Departamento de Salud del Gobierno de Navarra, y dentro de la Red Europea de Proyectos Piloto de Programas de «screening» de Cáncer de Mama. La condición de "participantes» la detentan mujeres de entre cuarenta y cinco y sesenta y cinco años que, siendo citadas por la correspondiente Consejería, decidieron colaborar con el programa. En el otro extremo, las «no participantes» son mujeres que, siendo citadas, no acudieron a hacerse la prueba mamográfica.

La muestra incluyó a mujeres de las distintas zonas geográficas de Navarra, esto es, Pamplona y comarca, Montaña, Media y Ribera. En lo que concierne a la edad, la muestra tenía una media de edad de las "participantes» de 56,5 años (desviación típica $=6,0$ ) y la de las «no participantes» de 58,1 años (desviación típica $=6,4)$. Las 512 mujeres "participantes» habían pasado un "screening" previo. Las 196 mujeres «no participantes» habían rechazado tomar parte en él.

El muestreo fue aleatorio a partir de los listados del Programa de Cáncer de Mama del Departamento de Salud del Gobierno de Navarra. El universo comprendió a mujeres empadronadas en Navarra con una edad entre cuarenta y cinco y sesenta y cinco años, esto es, a 60.908 mujeres. La muestra se estableció, por una parte, siguiendo proporcionalmente el número de participantes en cada una de las cuatro zonas geográficas en que se dividió Navarra y, por otra, atendiendo a cuotas de número de habitantes, ya que se partía de la hipótesis de que el número de habitantes podía hacer variar la participación o su rechazo. Para un nivel de confianza del 95 por 100, el margen de error es del \pm 3,6 por 100 para los datos globales, del $\pm 4,3$ por 100 para el grupo de "participantes», y, finalmente, del $\pm 6,8$ por 100 para las «no participantes».

La participación en la investigación fue voluntaria. Previamente cada persona de la muestra había recibido una carta personal con información acerca del objetivo del estudio. Posteriormente, se concertaba por teléfono una cita para la entrevista personal con cada una de las mujeres de la muestra.

\subsection{Variables}

Se incluyeron dos tipos generales de variables: $a)$ variables sobre actitudes hacia la salud, $\mathrm{y} b$ ) variables sociodemográficas.

a) Actitudes hacia la salud. Se evaluaron empleando la Escala de actitudes hacia la Salud de Rosenstock (1974). Este cuestionario se basa teóricamente en el 
modelo de creencias de salud de este mismo autor (Becker y Maiman,1977; Rosenstock, 1966, 1974). Las tres variables más relevantes de este modelo son: (1) Susceptibilidad: se refiere a la creencia de las personas acerca de la probabilidad de experimentar una condición potencialmente perjudicial; (2) Severidad: concierne a la percepción de amenaza para la propia persona, y (3) Beneficios: efectividad de conductas específicas para reducir la amenaza de la condición. La Escala de Actitudes hacia la Salud utilizada consta de nueve ítems que se cuantifican según una escala, tipo Likert, que oscila entre 1 y 5 , considerándose $1=$ muy en desacuerdo y $5=$ muy de acuerdo. Se han obtenido buenos niveles de fiabilidad y validez con la versión original del cuestionario (Champion, 1984). Para la presente investigación se aplicó la versión castellana del cuestionario, sobre la cual se han presentado datos apropiados de fiabilidad (consistencia interna) y validez convergente y discriminante (Lostao 1998, Lostao et al.,1995).

b) Variables sociodemográficas. Las variables sociodemográficas estudiadas fueron: edad, nivel de status social, nivel asociativo y estado civil. Los indicadores de status social y nivel asociativo se construyeron mediante las siguientes variables:

Indicador de status social: Se construyó a partir de las variables «nivel de estudios de la entrevistada", "profesión del cabeza de familia", "equipamiento del hogar» y «hábitos del hogar». Se establecen cinco niveles: alto, medio-alto, medio, medio-bajo y bajo.

Indicador de nivel asociativo: Se construyó a partir de las siguientes variables: "pertenencia a asociaciones» (clubs deportivos, piscinas, clubs culturales, corales, ateneos, casinos, juntas de vecinos, partidos políticos, sindicatos, otras asociaciones); "grado de participación en la vida social", contestación a tres preguntas sobre relaciones sociales (amistades, horas pasadas al día con personas no pertenecientes al núcleo familiar, satisfacción con el nivel de vida social fuera del hogar), y, finalmente, "profesión de la entrevistada». Los niveles resultantes fueron tres: alto, medio y bajo.

Todas las variables sociodemográficas fueron evaluadas mediante una entrevista estructurada, construida según los modelos desarrollados por el Centro de Investigación y Estudios Sociales (CIES). La experiencia del CIES en la elaboración de cuestionarios de salud dirigidos a distintos colectivos (tales como el Cuestionario de Salud de la Comunidad de Navarra, 1989, 1991) resultó particularmente relevante para la metodología de nuestra investigación.

\subsection{Tratamiento estadístico de los datos}

El tratamiento estadístico básico consistió en la realización de análisis de regresión logística y en el cálculo de los odds ratio (OR). El análisis de regresión logística es un método de análisis estadístico que permite describir la relación 
entre un conjunto de variables independientes y una variable dependiente dicotómica (Kleinbaum, 1994). En nuestro caso, la variable dependiente (dicotómica) fue la participación en el «screening» (participación=1, no participación=0).

En esta investigación, el análisis de regresión logística describe la asociación entre un conjunto de variables (factores de riesgo) y la probabilidad de participar o no en un programa de «screening» de cáncer de mama. Los parámetros del modelo de regresión logística tienen una interpretación sencilla en términos de OR (odds ratio). Éstos indican cuánto más probable (o verosímil) es la aparición de una respuesta $\mathrm{y}=1$ entre los casos que presentan $\mathrm{x}=1$ que entre los que presentan $\mathrm{x}=0$.

Como nosotros estábamos interesados en conocer la contribución relativa de las variables del modelo de creencias de salud y de las variables sociodemográficas en la predicción de la participación en el «screening», realizamos, primeramente, un análisis de regresión logística simple, con la variable dicotómica "participación» vs. «no participación» como variable dependiente (Kleinbaum, 1994), y las variables del HBM (health belief model) y las variables sociodemográficas separadamente como variables independientes. Posteriormente, se realizaron análisis de regresión logística múltiple donde se incluyeron todas las variables independientes de forma conjunta para observar el posible efecto de todas estas variables sobre la participación en el «screening».

Una forma adecuada de analizar las variables sociodemográficas es incluir en el modelo logístico variables indicadoras que representan las distintas categorías de la variable. El método más usado para el análisis de una variable nominal con $\mathrm{K}$ categorías es el denominado codificación de la categoría de referencia. Este método se basa en la construcción de $\mathrm{K}-1$ variables indicadoras, que son las que se incluyen en el modelo.

Se efectuaron análisis de regresión logística simple para evaluar la asociación entre factores potencialmente relativos a la participación en la prueba mamográfica utilizando chi-cuadrado $\left(\chi^{2}\right)$ para variables categóricas. La magnitud de la asociación (OR, odds ratio) e intervalos de confianza es calculada también para estas variables. Para el análisis de regresión logística se empleó el método forward stepwise, en el cual las variables van entrando o saliendo del modelo de una en una, a partir de un modelo inicial en el que únicamente figura la constante. Se utilizaron para la realización de dichos análisis los paquetes estadísticos SPSS for Windows (SPSS Inc., 1996).

\section{RESULTADOS Y DISCUSIÓN}

En las tablas 1 y 2 se muestran la distribución de factores asociados a la participación en el «screening» mamográfico de Navarra. En primer lugar, calculamos los índices de posibilidad de participación (OR) asociados a estas variables sociodemográficas, así como a las variables de creencias de salud. De acuerdo al análisis de regresión logística simple (tablas 1 y 2), la participación 
en un programa de «screening» está asociada significativamente a las variables sociodemográficas así como a variables de creencias de salud.

De todo el conjunto de estas variables, la participación en las pruebas mamográficas está fuertemente asociada con las personas más jóvenes de edad 45-50 ( $\mathrm{OR}=1,52)$; de estado civil casadas $(\mathrm{OR}=3,25)$; con nivel asociativo alto $(\mathrm{OR}=2,77)$; de status social alto $(\mathrm{OR}=5,13)$, así como con la variable severidad $(\mathrm{OR}=1,15)$. La participación en el «screening» no estaba significativamente asociada con la variable susceptibilidad y beneficios del modelo de creencias de salud. En estas dos variables no eran significativos los OR.

Los resultados del análisis de regresión logística múltiple están reflejados en la tabla 3. En el análisis de regresión logística múltiple, cuando todas las

\section{TABLA 1}

Análisis de regresión logística simple para las variables sociodemográficas (variables independientes) asociadas a la participación en el "screening" (variable dependiente) $(n=708)$

\begin{tabular}{|c|c|c|c|c|c|}
\hline & $\begin{array}{c}\text { Participantes } \\
(N=512) \\
(\%)\end{array}$ & $\begin{array}{c}\text { No participantes } \\
(N=196) \\
(\%)\end{array}$ & $\chi^{2}$ & $p$ & $I C(95 \%)$ \\
\hline \multicolumn{6}{|l|}{ Edad } \\
\hline 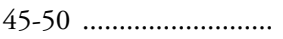 & 22,1 & 19,4 & & & $1,52(0,96-2,39)$ \\
\hline 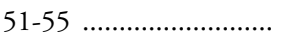 & 19,9 & 19,4 & & & $1,37(0,87-2,17)$ \\
\hline 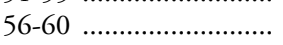 & 26,4 & 18,9 & & & $1,87(1,19-2,93)$ \\
\hline 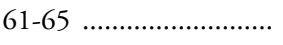 & 31,6 & 42,3 & $8,46^{*}$ & $<0,05$ & 1,00 \\
\hline \multicolumn{6}{|l|}{ Estado civil } \\
\hline 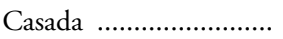 & 81,6 & 62,8 & & & $3,25(2,03-5,19)$ \\
\hline 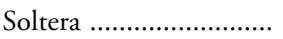 & 8,6 & 21,4 & & & $1,14(0,32-4,03)$ \\
\hline Viuda ............................... & 8,4 & 13,3 & & & $1,57(0,82-3,00)$ \\
\hline Separada .......................... & 1,2 & 2,6 & $31,14^{* * *}$ & $<0,01$ & 1,00 \\
\hline \multicolumn{6}{|l|}{ Nivel asociativo } \\
\hline 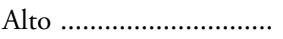 & 4,5 & 2,0 & & & $2,77(0,94-8,15)$ \\
\hline 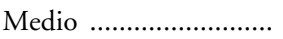 & 37,9 & 25,5 & & & $1,86(1,29-2,70)$ \\
\hline 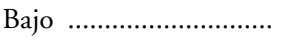 & 57,6 & 72,4 & $13,58^{* *}$ & $<0,01$ & 1,00 \\
\hline \multicolumn{6}{|l|}{ Status social } \\
\hline 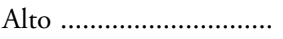 & 5,9 & 3,1 & & & $5,13(1,91-13,72)$ \\
\hline Medio-alto ...................... & 19,7 & 21,9 & & & $2,41(1,36-4,27)$ \\
\hline Medio ............................. & 38,5 & 33,2 & & & $3,11(1,83-5,27)$ \\
\hline Medio-bajo .................... & 28,5 & 21,9 & & & $3,48(1,98-6,11)$ \\
\hline 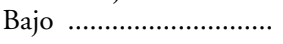 & 7,4 & 19,9 & $26,18^{* * *}$ & $<0,001$ & 1,00 \\
\hline $\begin{array}{r}* \mathrm{p}<0,05 \quad{ }^{* *} \mathrm{p}< \\
\text { NOTA: OR: odds ratio. } \\
\text { IC: intervalo de } \\
\%: \text { de participan }\end{array}$ & $\begin{array}{l}0,01 \\
\text { confianza, } 95 \\
\text { tes y no parti }\end{array}$ & $\begin{array}{l}\mathrm{p}<0,001 \\
\% \text {. } \\
\text { cipantes. }\end{array}$ & & & \\
\hline
\end{tabular}




\section{TABLA 2}

Análisis de regresión logistica simple para las variables del modelo de creencias de salud (variables independientes) asociadas a la participación en el "screening" (variable dependiente) $(n=708)$

\begin{tabular}{|c|c|c|}
\hline Variables del modelo de creencias de salud & $O R$ & $I C(95 \%)$ \\
\hline Severidad ............ & 1,15 & $1,11-1,20$ \\
\hline 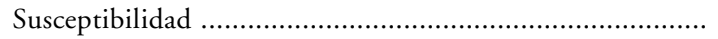 & 1,08 & $1,02-1,13$ \\
\hline Beneficios & 1,02 & $0,95-1,09$ \\
\hline
\end{tabular}

NOTA: OR: odds ratio.

IC: intervalo de confianza, 95\%.

\section{TABLA 3}

Análisis de regresión logistica múltiple para las variables del modelo de creencias de salud y variables sociodemográficas asociadas a la participación en el "screening"

$$
(n=708)
$$

\begin{tabular}{|c|c|c|c|c|c|c|c|}
\hline & $B$ & Wald & g.l. & $p$ & $R$ & $O R$ & IC (95\%) \\
\hline \multicolumn{8}{|l|}{ Severidad } \\
\hline Severidad ............ & 0,1463 & 44,3925 & 1 & 0,0000 & 0,2261 & 1,16 & $1,10-1,20$ \\
\hline \multicolumn{8}{|l|}{ Estado civil } \\
\hline Casada .................... & 0,6410 & 9,7100 & 1 & 0,0018 & 0,0964 & 1,90 & $1,26-2,84$ \\
\hline Soltera .................. & $-0,3239$ & 1,6547 & 1 & 0,1983 & 0,0000 & 0,72 & $0,44-1,18$ \\
\hline Viuda .................... & 0,0810 & 0,0916 & 1 & 0,7621 & 0,0000 & 1,08 & $0,64-1,83$ \\
\hline Separada ............... & & & & & & 1,00 & \\
\hline \multicolumn{8}{|l|}{ Nivel asociativo } \\
\hline Alto ...................... & 0,4628 & 1,35 & 1 & 0,2444 & 0,0000 & 1,58 & $0,72-3,46$ \\
\hline Medio ................... & 0,0786 & 0,1182 & 1 & 0,7310 & 0,0000 & 1,08 & $0,66-3,43$ \\
\hline Bajo ......................... & & & & & & 1,00 & \\
\hline \multicolumn{8}{|l|}{ Status social } \\
\hline Alto ...................... & 0,2530 & 0,4017 & 1 & 0,5262 & 0,0000 & 1,28 & $0,58-2,81$ \\
\hline Medio-alto ......... & $-0,0320$ & 0,0259 & 1 & 0,8721 & 0,0000 & 0,96 & $0,65-1,43$ \\
\hline Medio .................... & 0,3423 & 3,8363 & 1 & 0,0502 & 0,0471 & 1,40 & $0,99-1,98$ \\
\hline Medio-bajo ........ & 0,2883 & 2,2846 & 1 & 0,1307 & 0,0185 & 1,33 & $0,91-1,91$ \\
\hline Bajo ....................... & & & & & & 1,00 & \\
\hline
\end{tabular}

NoTA: OR: odds ratio.

IC: intervalo de confianza, 95\%. 
variables son incluidas en la ecuación, las variables que mejor predicen la participación en el «screening» son la severidad (variable del modelo de creencias de salud) $(\mathrm{OR}=1,16, \mathrm{p}<0,001)$ y las variables sociodemográficas relacionadas con el estado civil casada $(\mathrm{OR}=1,90, \mathrm{p}<0,01)$ y el status social medio $(\mathrm{OR}=1,40$, $\mathrm{p}=0,05)$. Las variables que no presentan asociación en este análisis y quedan fuera son la variable susceptibilidad, edad, nivel asociativo y beneficios.

Se ha constatado que el grado de conocimiento del cáncer de mama, la creencia acerca del pronóstico y el nivel de ansiedad ante esta enfermedad son factores que están relacionados con las conductas de participación en el «screening» de cáncer de mama (Lostao et al., 1996) y, por tanto, son variables que deben ser tenidas en cuenta para crear el perfil de las mujeres participantes, así como para explicar las diferencias sociales en los comportamientos de prevención de las mujeres en tales programas de «screening».

Algunos autores han señalado que la participación decrece conforme aumenta la edad (Van den Heuvel, 1977; Vermost, 1978; Rimer et al., 1992; King et al., 1993; Calle et al., 1993). También se ha referido que la participación es mayor en las mujeres casadas que en las solteras o separadas (Lostao et al., 1996; Rutter et al., 1993; Sutton et al., 1994). Asimismo, la mayor participación se ha relacionado con los niveles más elevados de nivel asociativo y de status social (Hobbs et al., 1980; Calnan, 1984; French et al., 1982; Ross et al., 1994; Urban et al., 1994; Breen and Kessler, 1994).

En nuestra investigación hemos estudiado las variables sociodemográficas de edad, estado civil, nivel asociativo y status social, así como también las variables vinculadas al modelo de creencias de salud. Los resultados basados en los análisis de regresión logística simple muestran una asociación positiva entre acudir a realizarse la mamografía y las variables mencionadas, así como también con la variable severidad. Los resultados muestran efectos significativos $(\mathrm{p}<0,05)$ de la variable edad, lo que sugiere una ligera asociación con la participación entre las edades más jóvenes. No obstante, en el análisis de regresión logística múltiple esta asociación desaparece para la variable edad. Asimismo, ciertas variables, como estar casada y pertenecer a niveles elevados de status social y asociatividad, parecen predecir la conducta de participación tal y como se desprende de nuestros análisis de regresión logística simples. Tras el análisis de regresión logística múltiple, no obstante, se constata que las únicas variables sociodemográficas que predicen la participación son ser una mujer casada y poseer un status social medio. Nuestros resultados se verían apoyados por los estudios de Van den Heuvel (1977) y Rutter et al. (1993), así como por los trabajos de Sutton et al. (1994), quienes ya habían establecido un mayor porcentaje de participación en el «screening» por parte de las mujeres casadas, debido en gran parte a un mayor apoyo familiar, que favorecía su implicación en programas de salud preventivos. Igualmente, nuestros datos coinciden con los resultados obtenidos por Vermost (1978) que mostraban reducidos niveles de participación en los estratos sociales más bajos y en los más altos, siendo, por el contrario, la participación más elevada entre las mujeres de clase media. 
Bowling (1989) ha subrayado como explicación de este resultado el que las creencias, percepciones y conocimientos (la "cultura») de las personas de clase media tienden a ser más coincidentes con la aproximación científico-médica a los problemas de la salud, mostrando, además, los pacientes de clase media una actitud de mayor confianza en la comunicación con sus doctores. El resto de las variables sociodemográficas no resultaron estadísticamente significativas.

En cuanto al papel de las variables incluidas en esta investigación derivadas del modelo de creencias de salud de Rosenstock (Rosenstock, 1966, 1974) y, en concreto, la variable severidad, se observa su asociación positiva con la conducta de participación en el «screening». Según establece el modelo de Rosenstock, se trata de actitudes hacia la salud que facilitan la realización de conductas encaminadas a la prevención de la enfermedad y mejora de la salud.

Diferentes autores como Owens et al. (1987) y Wardle y Pope (1992) ya habían subrayado la importancia de las creencias de salud en relación con la participación en el «screening». Estos autores sugieren que las creencias de salud de las mujeres que atienden el «screening» difieren de las mujeres que no participan en él. Estas mujeres tienen diferentes creencias acerca de la salud y acerca de la conducta de salud preventiva. Rutledge et al. (1988) analizó lo que ha podido influir en la participación o no participación de mujeres en un «screening» mamográfico, según sus «opiniones sobre la salud» (modelo de creencias de salud), y señaló que las participantes se sentían, significativamente, más amenazadas por el cáncer de mama. El modelo de creencias de salud ha sido aplicado también por varios autores a contextos preventivos, algunos de ellos semejantes, otros diferentes del que nosotros hemos empleado (Janz y Becker, 1984). Entre otros ámbitos de salud en los que se ha aplicado dicho modelo cabe citar las conductas adictivas y el SIDA (Strecher et al.,1997), la autoexploración para el cáncer de mama (Calnan and Rutter, 1986; Champion, 1990,1997), la participación en chequeos dentales (Chen y Land, 1986, 1990), la utilización de servicios sanitarios (Leavitt,1979; Reese y Smith,1997) y el cumplimiento de prescripciones terapéuticas (Becker et al.,1972; Becker y Rosenstock, 1978).

Los resultados obtenidos permiten comprobar que las participantes y no participantes parecen diferenciarse de forma muy clara con respecto a la variable severidad. Estos resultados coinciden igualmente con los hallados por Rutter, Quine y Chesham (1993), los cuales señalaron que la severidad percibida era el más importante predictor de la conducta preventiva de participación.

Según las investigaciones de Rosenstock, y en relación a las creencias de salud, existe una postura unitaria apoyada por trabajos de diferentes autores referidos a comportamientos preventivos de enfermedad en sujetos asintomáticos (Hochbaum, 1956; Kegeles, 1963; Leavitt, 1979). Estos autores señalan que las creencias de salud son necesarias para pasar tests preventivos o de «screening». Esta línea de investigación confirma los resultados obtenidos acerca de que los individuos buscan atención sanitaria y se preocupan por la prevención cuando ellos creen que una enfermedad es seria. 
El modelo de creencias de salud es ampliamente aplicado hoy como una explicación de la conducta preventiva de salud individual, así como para explicar otros problemas de salud pública existentes en nuestra sociedad actual, como conductas de consumo de alcohol (Minugh et al., 1998), conductas relacionadas con enfermedades como el sida (Strecher et al., 1997) y para ayudar en la detección precoz del cáncer de mama mediante el «screening» mamográfico (Thomas et al., 1996; Savage y Clarke,1996; Champion, 1997). Así, también en los trabajos de Champion (1984, 1990, 1997), donde se trata de identificar variables actitudinales especificadas por el modelo de creencias de salud relativas en la frecuencia de conductas preventivas de autoexploración, los resultados apoyan la utilización de estas variables actitudinales para predecir conductas actuales de las mujeres en relación a la autoexploración de las mamas.

En este sentido, la variable severidad podría ser considerada como un importante predictor del comportamiento de participación en el «screening». La variable severidad tiene un efecto independiente, es decir, predice la participación de forma independiente. No obstante, el valor de los odds ratio de esta variable, aun siendo importante, no es excesivamente alto. Consideramos que la variable es suficientemente válida en su relación con la participación, ya que los datos obtenidos permiten afirmar que, efectivamente, esta variable junto con las variables estado civil casada y status social medio predicen de forma estadísticamente significativa la participación de las mujeres en el «screening». El modelo de creencias de salud muestra su poder para explicar la influencia de las dimensiones socioculturales en las prácticas relativas a la salud preventiva, en concreto, en la posibilidad de realizar pruebas mamográficas preventivas, apoyando la idea de que las personas tienen sus propias creencias sobre lo que es relevante respecto a su propia salud.

Los comportamientos preventivos de la población no parecen estar explicados por un conjunto único de razones. El que la población lleve a cabo comportamientos preventivos va a depender en gran medida de la percepción que las personas tienen de la amenaza que dicha enfermedad constituye para ellas y de la eficacia que cree que el llevar a cabo esa conducta tendrá para reducir la amenaza. Las creencias son muy importantes en las conductas de salud; en este caso, en la conducta preventiva y, a su vez, dependen de los sistemas socio-culturales de pertenencia y de referencia. La probabilidad de que la población lleve a cabo una conducta de salud preventiva dada está determinada, por otra parte, por la evaluación que la persona hace de dicha conducta de salud en términos de su viabilidad y eficacia (es decir, la estimación subjetiva de los beneficios potenciales de la acción para reducir la severidad o gravedad), contrapesada con las percepciones de los costos físicos, sociales, económicos y de otro tipo (barreras) implicadas en la acción preventiva.

Una de las limitaciones señaladas a este tipo de estudios hace referencia a que la mayoría de los estudios realizados sobre la eficacia explicativa del HBM son retrospectivos, y, por tanto, resulta imposible determinar a partir de ellos, 
si la creencia de salud existía antes o se generó después de la realización de la conducta de salud estudiada. Sin embargo, la hipótesis de que la conducta de salud preventiva está determinada por una constelación de creencias sólo puede ser comprobada adecuadamente cuando se sabe que las creencias existían antes de la conducta que se supone que determinan. Otra limitación de este estudio es que los resultados de esta investigación han sido los obtenidos dentro del programa piloto en España, llevado a cabo en Navarra, por lo que habrá que ver en la sucesiva implantación de estos programas en las distintas Comunidades Autónomas qué ocurre a este respecto.

Distintos estudios han demostrado que los servicios de salud preventivos son utilizados, en mayor medida, por personas de status socieconómico alto (Dutton, 1978, 1979, 1986; Siegrist, 1989). Las personas que tienen más recursos en términos de conocimientos, dinero, poder, prestigio y relaciones sociales parecen estar mejor equipadas para evitar los riesgos y adoptar las estrategias preventivas que están disponibles en un momento y en un lugar determinados. Obviamente, antes del descubrimiento del tipo de «screening" mamográfico estaba claro que no existía ninguna relación entre éste y el indicador de status socieconómico, así como tampoco con la relación de dicho status y mortalidad. Sin embargo, una vez descubiertas innovaciones beneficiosas como éstas, que necesitan agentes individuales y acceso a recursos sanitarios, llegaran a asociarse (una vez aplicados) con el status socieconómico, por lo que desempeñará un papel destacado en los gradientes de mortalidad de esta enfermedad. Diferentes estudios relacionan la participación en los «screenings» con el nivel de titulación y los ingresos familiares. No es el caso de este estudio, ya que se observó una elevada presencia de mujeres con niveles educativos bajos.

La implantación extendida del «screening» mamográfico en España es demasiado reciente para que haya logrado un impacto total sobre la mortalidad debida a esta enfermedad. Las acciones de implantación de «screenings», si bien todavía no han dado lugar a desigualdades sociales de mortalidad en España, es previsible que acaben haciéndolo.

El impacto global de los «screenings» ha sido evitar fallecimientos. Ésta es la consecuencia deseada por los implementadores del desarrollo y puesta en marcha de los «screenings». Como señala Robert Merton, estas acciones deliberadas también presentan consecuencias imprevistas de la acción social. Una razón es que ninguna acción se produce en un "vacío social», lo que quiere decir que sus efectos probablemente tengan ramificaciones en otras esferas de valor e interés (Merton, 1936). Cuando innovaciones beneficiosas como la puesta en marcha de un "screening" preventivo se desarrollan en nuestra sociedad, su puesta en práctica se produce necesariamente en el contexto de desigualdades en las áreas de conocimiento, dinero, poder y relaciones sociales. Estas desigualdades determinan la distribución del beneficio sanitario, y al hacerlo tienen un impacto sobre los patrones de mortalidad que ni los promotores ni los que llevan a cabo la implantación de esa innovación desean. Aunque estas consecuencias puedan parecer inevitables, los niveles de desigualdad 
social en materia de salud son sensibles a las políticas llevadas a cabo en esta área. De ahí la importancia de incluir estas variables y otras relacionadas con éstas a la hora de llevar a cabo la implementación masiva en todo el territorio español.

La importancia de las variables y dimensiones subyacentes a las conductas de respuesta ante los programas de «screening» poblacionales ha crecido de forma considerable. El conocimiento de los factores de riesgo de origen medioambiental, así como las medidas tendentes a su detección sobre la base de los primeros síntomas ocupan un lugar destacado en las campañas para combatir dicha enfermedad. Las campañas preventivas suelen tener carácter público en sociedades como las europeo-occidentales, caracterizadas por Estados de bienestar altamente desarrollados. Sociedades cuyas instituciones públicas garantizan el derecho social "a la protección de la salud", con el concurso de combinaciones varias de agentes públicos y privados, pero con primacía clara de los primeros. Son, por lo común, las autoridades sanitarias las iniciadoras o, en otros casos, las encargadas de la implementación de programas y campañas orientadas al ejercicio efectivo de ese nuevo derecho incorporado a los textos constitucionales elaborados tras la Segunda Guerra Mundial. Por su parte, las autoridades sanitarias deberían mejorar las medidas de implementación beneficiándose de la investigación científico-social, para seguir aumentando los niveles de participación, habida cuenta del enorme beneficio obtenible con costes sólo marginalmente más elevados.

\section{CONCLUSIÓN}

El cáncer de mama es una enfermedad socialmente temida y una de las primeras causas de mortalidad en la población femenina de los países industrializados. Asumiendo que las tasas de incidencia y mortalidad por cáncer de mama en España han crecido de forma alarmante, el establecer un diagnóstico precoz de la enfermedad es clave, ya que este diagnóstico está directamente relacionado con los índices de supervivencia y con la calidad de vida de las mujeres afectadas. Esto ha impulsado el desarrollo de programas de prevención poblacionales para dicha enfermedad. La evidencia científica a favor de la eficacia del «screening» mediante mamografía para detectar el cáncer de mama, ha sido señalada para las mujeres de grupos de edad de 45-65 años.

En la implantación y desarrollo de programas de «screening" masivos y voluntarios financiados por el Sistema de Salud Pública, hay que subrayar la importancia de realizar campañas diseñadas científicamente para conseguir altos índices de participación y que además tengan una adecuada capacidad de impacto. Para ello, es necesario un conocimiento de la naturaleza de las creencias de salud de la población, así como de las actitudes del público hacia las medidas preventivas que poseen capacidad predictiva independiente respecto a la participación de la población en dichos programas. Otras variables de interés se refieren al estado civil de las participantes y su status social, obteniéndose 
una participación mayor entre las mujeres casadas y de clase media, que tienden a beneficiarse de los servicios preventivos médicos ofrecidos por la red sanitaria pública en mayor medida que otros subconjuntos de población.

El modelo de creencias de salud constituye un marco que ayuda a comprender las variables que influyen en la utilización de los servicios de salud preventivos, con el objetivo de aumentar la participación de la población para que dichos programas sean rentables e intentar reducir los costes sanitarios derivados de una ineficaz utilización. Claramente, las actitudes humanas, las creencias y los valores juegan un papel determinante en la utilización de los servicios preventivos de salud. La Sociología de la Salud (Gochman, 1971; Becker et al., 1977; Dutton, 1978; Becker, 1979; Janz y Becker, 1984; Cockerham, 1998) da buena información en este plano, y posteriores investigaciones deberían incluir estas y otras variables relacionadas, dirigiendo los esfuerzos hacia una mejora de la prevención y de la salud comunitaria. La prevención es una práctica netamente social en tanto que tiene como objeto la sociedad, y a su vez supone la implicación de la población en las tareas de cuidado de la salud.

En suma, las variables que hemos evaluado son de particular interés para predecir la participación en el «screening». Así, la prevención efectiva del cáncer de mama y los programas de detección deberían tener en cuenta la importancia de este tipo de variables de creencias de salud además de las variables sociodemográficas para obtener resultados más o menos eficaces y para que la gente participe más activamente en campañas de «screening» de cáncer de mama.

\section{REFERENCIAS BIBLIOGRÁFICAS}

American Cancer Society (1996): "Cancer Facts and Figures-1996», The American Cancer Society, Atlanta.

AsCunCE, N. (1991): «Mortalidad por cáncer en España. Movimiento natural de la población española. Prevención secundaria del cáncer», en Jordi Estapé e I. Burgos (eds.), en Medicina General y Cáncer, Doyma, Barcelona, pp. 67-75.

Ascunce, N.; Del Moral, A.; Murillo, A.; Alfaro, C.; Apesteguia, L.; Ros, J., et al. (1994): «Early detection programme for breast cancer in Navarra (Spain)», en European Journal Cancer Prevention, 3, pp. 41-48.

Ascunce, N.; González, J.; González, A.; Herranz, C.; Marqués, A., y Martín, J. (1993): "Criterios generales y recomendaciones para la elaboración de programas de detección precoz de cáncer de mama y cáncer de cérvix uterino en España», en Revista de Sanidad e Higiene Pública, 67, pp. 23-37.

BowLING, A. (1989): «Implications of preventive health behaviour for cervical and breast cancer screening programmes: A review», en Family Practice, 6, pp. 224-231.

Becker, M. H.; Drachman, R. H., y KirSCHT, J. P. (1972): «Predicting mothers'compliance with pediatric medical regimens», en Medical Care, 81, pp. 843-854.

Becker, M. H., y MAIMAN, L. A. (1975): «Sociobehavioural determinants of compliance with health and medical care recommendations», en Medical Care, 13, pp. 10-24. 
BECKER, M. H. (1974): The health belief model and personal health behavior, San Francisco, Society for Public Health Education, Inc.

- (1977): «Mothers' health beliefs and children's clinic visits», en Journal of Community Health, 3, pp. 125-135.

- (1979): «Psychosocial Aspects of Health-Related Behavior», en Handbook of medical socio$\log y, 3^{\text {th }}$ ed., H. Freeman, S. Levine y L. Reeder (eds.), pp. 253-274, Englewood Cliffs, N.J.: Prentice Hall.

Becker, M. H., y Rosenstock, I. M. (1978): "Compliance with a medical regimen for asthma: A test of the health belief model", en Public Health Reports, 93, pp. 268-277.

- (1989): «Health promotion, disease prevention, and programme retention», en Handbook of medical sociology, $4^{\text {th }}$ ed., H. Freeman y S. Levine (eds.), pp. 284-305, Englewood Cliffs, N.J.: Prentice Hall.

Becker, M. H.; Maiman, L. A.; Kirscht, J. P.; Haefner, D. P., y Drachman, R. H. (1977): "The health belief model and prediction of dietary compliance: a field experiment», en Journal of Health and Social Behavior, 18, pp. 348-366.

Blamey, R. W.; Wilson, A. R. M.; PATniCK, J., y DixON, J. M. (1994): «Screening for breast cancer», en British Medical Journal, 309, pp. 1076-1079.

Bonfill, X.; Marzo, M. M.; Medina, C.; Roura, P., y Rué, M. (1992): «L'efectivitat del cribatge del cáncer de mama en el nostre entorn", en Gaceta Sanitaria, 6, pp. 128-142.

Borrás, J. M.; Ciurana, R., y Marzo, M. M. (1994): "Cribado del cáncer de mama», en Medicina Clínica, 102, supl. 1, pp. 70-75.

Boyle, P.; Veronesi, V.; Tubiana, M.; Alexander, F. E.; Calais, D. A.; Silva, F.; Denis, L. J. et al. (1995): European School of Oncology. Advisory Report to the European Commission for the "Europe Against Cancer Programme». European Code Against Cancer, en European Journal Cancer, 31A, pp. 1395-1405.

BREEN, N., y KeSSLER, L. (1994): "Changes in the use of screening mammography: evidence from the 1987 and 1990 national health interview surveys», en American Journal of Public Health, 84, pp. 62-67.

Brown, C. M., y SEgAL, R. (1996): "Ethnic differences in temporal orientation and its impliccations for hypertension management», en Journal of Health and Social Behavior, 37, pp. 350-361.

Calle, E. E.; Flanders, W. D.; Thun, M. J., y Martin, L. M. (1993): «Demographic predictor of mammography and pap smear screening in US women", en American Journal of Public Health, 83, pp. 53-60.

CALNAN, M. (1984): "The health belief model and participation in programmes for the early detection of breast cancer: a comparative analysis», en Social Science and Medicine, 19, pp. 823-830.

Calnan, M., y RutTer, D. R. (1986): «Do Health Beliefs predict Health Behaviour? An Analysis of Breast Self-Examination", en Social Science and Medicine, 22, pp. 673-678.

Champion, V. L. (1984): «Instrument development for health belief model constructs», en Advances in Nursing Science, 6, pp. 73-85.

- (1990): «Breast Self-Examination in women 35 and older: a Prospective Study», en Journal of Behavioral Medicine, 3, pp. 523-538.

- (1993): «Instrument refinement for breast cancer screening behaviours», en Nursing Research, 42, pp. 139-143.

ChAmpion, V. L., y SCOTT, C. R. (1997): "Reliability and validity of breast cancer screening belief scales in African American women», en Nursing Research, 46, pp. 331-337.

Chen, M., y LAND, K. C. (1986): «Testing the Health Belief Model: LISREL analyses of alternative models of causal relation ships bet ween health beliefs and preventive dental behavior", en Social Psychology Quarterly, 49, pp. 56-60.

- (1990): «Socioeconomic status (SES) and the Health Belief Model: LISREL analysis of unidimensional versus multidimensional formulations», en Journal of Social Behavior and Personality, 5, pp. 263-284. 
Cockerham, W. C. (1998): «Health Behavior», en Medical Sociology, $7^{\text {th }}$ ed., W. C. Cockerham (ed.), pp. 84-101, New Jersey, Prentice-Hall.

Cockerham, W. C.; Gerhard, K., y Lueschen, E. (1988a): «Social stratification and health lifestyles in two systems of health caredelivery: A comparison of America and West Germany", en Journal of Health and Social Behavior, 29, pp. 113-126.

Coleman, M. P.; Esteve, J.; Damiecki, P.; Arslan, A., y Renard, H. (1993): «Trends in cancer incidence and mortality», Scientific Publication, núm. 121, Lyon, International Agency for Research on Cancer.

De WaARd, F. (1993): «Breast Cancer: the scene in Europe», en A. Gad y M. Rosselli del Turco (eds.), Breast Cancer Screening in Europe, pp. 3-5, Berlín, Springer-Verlag.

Donato, F.; Bollani, A.; Spiazzi, R.; Soldo, M.; Pasquale, L.; MonarCa, S., et al. (1991): "Factors associated with non-participation of women in a breast cancer programme in a town in Northern Italy", en Journal of Epidemiology and Community Health, 45, pp. 59-64.

Donovan, D.; Middleton, J., y Ellis, D. (1988): «Early detection of breast cancer», en Lancet, 1, p. 685.

Dood, G. D. (1992): «American Cancer Society Guidelines on Screening for Cancer: An Overview», en Cancer, 42, pp. 177-80.

DutTon, D. B. (1978): «Explaining the low use of health services by the poor: cost, attitudes, or delivery sistems?», en American Sociological Review, 43, pp. 348-368.

- (1979): "Patterns of ambulatory health care in five different delivery systems», en Medical Care, 17, pp. 221-241.

- (1986): «Social class, health and illness», pp. 31-62, en Applications of social science to clinical medicine and health policy, L. Aiken y D. Mechanic (eds.), New Brunswick, N.J., Rutgers University Press.

EARDlEY, A., y ElKIND, A. (1990): "A pilot study of attendance for breast cancer screening», en Social Science and Medicine, 30, pp. 693-699.

FinK, R.; SHAPIRO, S., y LEVINSON, J. (1968): «The reluctant participant in a breast screening programme», en Public Health Reports, 83, pp. 479-490.

FinK, R.; SHAPIRO, S., y ROESTER, R. (1972): «Impact of efforts to increase participation in repetitive screenings for early breast cancer detection", en American Journal of Public Health, 62, pp. 328-336.

Fletcher, S. W.; Black, W.; Harris, R.; Rimer, B. K., y Shapiro, S. (1993): «Report of the International Workshop on screening for breast cancer», en J. Natl. Cancer Inst., 85, pp. 1644-1656.

French, K.; Porter, A. M.; Robinson, S. E.; McCallum, F. M.; Howie, J. R., y Roberts, M. M. (1982): "Attendance at a breast screening clinic: a problem of administration or attitudes», en British Medical Journal, 285, pp. 617-620.

García Arcal, M. D.; Pollán, M., y López-Abente, G. (1994): «Mortalidad por cáncer de mama en la Comunidad Europea (1970-1985)», en Medicina Clínica, 102, pp. 125-128.

Gochman, D. S. (1971): «Some correlates of children's health care beliefs and potential behavior", en Journal of Health and Social Behavior, 12, pp. 148-154.

Hobbs, P.; Smith, A.; George, W. D., y Sellwood, R. A. (1980): «Acceptors and rejectors of an invitation to undergo breast screening compared with those who referred themselves», en Journal of Epidemiology and Community Health, 34, pp. 19-22.

Hochbaum, G. M. (1956): "Why people seek diagnostic X-Rays», en Public Health Reports, 71, pp. 377-380.

INDICADORES DE SALUD: Segunda evaluación en España del programa regional europeo Salud para todos, p. 131, Ministerio de Sanidad y Consumo, Secretaría General de Salud, Madrid, 1993.

Janz, N. K., y BeCKer, M. H. (1984): «The Health Belief Model: a decade later», en Health Education Quarterly, 11, pp. 1-47.

Kegeles, S.S. (1963): «Why people seek dental care: A test of a conceptual formulation», en Journal of Health and Human Behavior, 4, pp. 166-173. 
Kegeles, S. S. (1980): "The Health Belief Model and Personal Health Behavior», en Social Science and Medicine, 14C, pp. 227-229.

Kerlikowske, K.; Grady, D.; Rubin, S. M.; SAndrock, C., y Ernster, V. L. (1995): «Efficacy of screening mammography: a meta-analysis», en Journal of American Medical Association, 273, pp. 149-154.

Kessler, L. G.; Feuer, E. J., y Brown, M. L. (1991): «Projections of the breast cancer burden to U.S. women: 1990-2000", en Preventive Medicine, 20, pp. 1386-1388.

King, E. S.; Resch, W.; Rimer, B., y Lerman, C. (1993): «Breast Cancer Screening practices among retirement community women», Preventive Medicine, An International Journal Devoted to Practice and Theory, 22, pp. 1-19.

KirsCHT, J. P. (1974): "The health belief model and illness behavior», en Health Education Monographs, 2, pp. 387-408.

Kleinbaum, D. G. (1994): "Logistic Regression: a Self-Learning Text», Statistics in the Health Sciences, Springer-Verlag, New York.

Knight, R. A., y HaY, D. A. (1989): "The relevance of the health belief model to Australian smokers", en Social Science and Medicine, 28, pp. 1311-1314.

LEAVITT, F. (1979): "The health belief model and utilization of ambulatory care services», en Social Science and Medicine, 18, pp. 105-112.

Link, B. G.; Northridge, M. E.; Phelan, J. C., y Ganz, M. L. (1998): «Social Epidemiology and the Fundamental Cause Concept: On the Structuring of Effective Cancer Screens by Socioeconomic Status», en The Milbank Quarterly, 76, 3, pp. 375-402.

Lostao, L.; Joiner, T. E.; Pettit, J. W.; Chorot, P., y Sandin, B. (2001): «Health Beliefs and Illness Attitudes as Predictors of Breast Cancer Screening Attendance», en European Journal of Public Health (en prensa).

LOSTAO, L. (1998): "Health inequalities and participation in a breast cancer screening program», 7. European Congress of the European Society of Health and Medical Sociology, Rennes, August 1998 , p. 259.

Lostao, L.; Chorot, P.; SAndin, B., y LACABE, F. (1996): «Variables psicosociales relacionadas con la participación en un "screening" de cáncer de mama", en Arbor, 608, pp. 33-71.

Lostao, L.; Sandin, B.; Chorot, P., y Lacabe, F. (1995): "Anxiety, coping and health beliefs as predictor variables of attending for breast screening». $24^{\text {th }}$ Congress of the European Association for Behavioural and Cognitive Therapies. Corfú (Greece), September 1995 , p. 248.

luengo, S.; Lazaro, P.; Madero, R.; Alvira, F.; Fitch, K.; Azcona, B.; Pérez, J. M., y Caballero, P. (1996): "Equity in the access to mammography in Spain», en Social Science and Medicine, 43, pp. 1263-1271.

Luengo, S.; Azcona, B.; LÁzaro, P., y Madero, R. (1997): «Programas de detección precoz del cáncer de mama y acceso a la mamografía en España", en Medicina Clínica, 108, pp. 761-766.

MCKINLAY, J. B. (1972): «Some approaches and problems in the study of the use of services. An overview», en Journal of Health and Social Behavior, 13, pp. 115-152.

MERTON, R. K. (1936): "The unanticipated consequences of purposive social action», en American Sociological Review, 1, pp. 894-904.

Minugh, P. A.; Rice, C., y Young, L. (1998): "Gender, health beliefs, health behaviors, and alcohol consumption", en American Journal of Drug and Alcohol Abuse, 24, pp. 483-497.

Morrison, A. S. (1993): «Screening for cancer of the breast», en Epidemiological Review, 15, pp. 244-255.

Owens, R. G.; Daly, J.; Heron, K., y Leinster, S. J. (1987): «Psychological and Social Characteristics of Attenders for Breast Screening», en Psychology and Health, 1, pp. 303-313.

PAS, L., y Robra, B. P. (1993): "Overview of European Screening Programmes», en A. Gad y M. Rosselli del Turco (eds.), Breast Cancer Screening in Europe, pp. 7-13, Berlín, SpringerVerlag.

Programa «Europa contra el cáncer» (1986): Propuesta de plan de acción 1987-1989. Comisión de las Comunidades Europeas, Bruselas, 16 de diciembre de 1986. 
ReESE, F. L., y SMITH, W. R. (1997): «Psychosocial determinants of health care utilization in sickle cell disease patients", en Annals of Behavioral Medicine, 19, pp. 171-178.

Rimer, B. K.; Ross, E.; Cristinzio, C. S., y KING, E. (1992): «Older women’s participation in breast screening», Forum of Breast Cancer Screening in older women, en Journal of Geronthology, 47, pp. 85-91.

Rodríguez, C.; Plasencia, A.; Ferrer, F., y Gómez, A. (1991): «Programa PAM de detección temprana de cáncer de mama: primeros resultados (1987-1990)», en Medicina Clínica, 96, pp. 206-210.

Rodríguez, C.; Plasencia, A., y Schroeder, D. G. (1995): «Predictive factors of enrollmen and adherence in a breast cancer screening programme in Barcelona (Spain)", en Social Science and Medicine, 40, pp. 1155-1160.

Romans, M. C. (1993): «Utilization of Mammography Social and Behavioral Trends», en Cancer, 72, pp. 1475-1477.

Rosenblatt, D., y Suchman, E. A. (1964): «Blue-collar Attitudes and Information towards Health and Illness», pp. 324-333, en Studies of the American Worker, edited by A. Shostak y W. Gomberg, Englewood Cliffs, NJ, Prentice-Hall.

Rosenstock, I. (1966): «Why people use health services», en Milbank Memorial Fund Quarterly, 44, pp. 94-127.

- (1974): «The Health Belief Model and preventive health behavior», en Health Education Monographs, 2, pp. 354-386.

Ross, N. A.; Rosenberg, M. W.; Pross, D. C., y Bass, B. (1994): "Contradictions in women's health care provision: A case study of attendance for breast cancer screening», en Social Science and Medicine, 39, pp. 1015-1025.

Rutledge, D. N.; Hartmann, W. H.; Oney, K. P., y Winfield, A. C. (1988): «Exploration of Factors Affecting Mammography Behaviors», en Preventive Medicine, 17, pp. 412-422.

Rutter, D. R.; Quine, L., y Chesham, D. J. (1993): Social Psychological Approaches to Health, New York, Harvester.

SaVAGe, S. A., y Clarke, V. A. (1996): "Factors associated with screening mammography and breast self-examination intentions", en Health Education Research, 11, pp. 409-421.

Segura, J. M.; Porta, M.; Macia, F.; Casamitjana, M., y Castells, X. (1998): «Factores condicionantes de la cobertura, la respuesta y la participación en un programa de cribado del cáncer de mama", en Medicina Clínica, 111, 251-256.

Shapiro, S.; StraX, P., y Venet, L. (1971): «Periodic Breast Cancer Screening in Reducing Mortality from Breast Cancer", en Journal of the American Medical Association, 215, pp. 1777-1785.

Siegrist, J., y Bertram, H. (1970, 1971): «Schichtspezifische variationen des Krankheitsver haltens", Soziale Welt (20/21), pp. 206-218.

SIEGRIST, J. (1989): «Steps towards explaining social differentials in morbidity: the case of West Germany», pp. 353-371, en Health inequalities in European countries, J. Fox (ed.) Aldershot, U.K., Gower.

Smith, R. A. (1992): «Breast Cancer Screening Guidelines», en Women's Health Issues, 2, pp. 212-217.

SPSS Inc. (1996): SPSS Base 7.0 for Windows. User's Guide. SPSS Chicago Inc.

Strecher, V. J.; Champion, V. L., y Rosenstock, I. M. (1997): «The health belief model and health behavior", pp. 71-91, en Handbook of Health Behavior Research 1: Personal and social determinants, D. S. Gochman (ed.) New York, Plenum Press.

Sutton, S.; Bickler, G.; SANChO-Aldridge, J. P., y SAidi, G. (1994): «Prospective study of predictors of attendance for breast screening in London", en Journal of Epidemiology and Community Health, 48, pp. 65-73.

Taplin, S.; Anderman, C., y Grothaus, L. (1989): «Breast cancer risk and participation in mammographic screening», en American Journal of Public Health, 79, pp. 1494-1498.

Thomas, L. R.; Fox, S. A.; Leake, B. G., y Roetzheim, R. G. (1996): "The effects of health beliefs on screening mammography utilization among a diverse sample of older women», en Women and Health, 24, pp. 77-94. 
Urban, N.; Anderson, G. L., y Peacock, S. (1994): «Mammography screening: how important is cost as a barrier to use?», en American Journal of Public Health, 84, pp. 50-55.

VAN DEN Heuvel, W. J. A. (1978): «Participants and non-participants in a mammography Mass-screening: who is who?», en Breast Cancer (Edited by Brand, P. C., y van Keep, P. A.), pp. 97-103, M.T.P. Press, Lancaster.

Vermost, L. (1978): «Factors affecting participation in Cancer Screening pro Programmes», en Breast Cancer (Edited by Brand, P.C., y van Keep, P. A.), pp. 91-96. M.T.P. Press, Lancaster.

Vernon, S. W.; LaVille, E. A., y Jackson, G. L. (1990): "Participation in breast screening programmes: a review», en Social Science and Medicine, 30, pp. 1107-1118.

WARDlE, J., y POPE, R. (1992): "The psychological costs of screening for cancer», Invited Review, en Journal of Psychosomatic Research, 36, pp. 609-624.

White, E.; Urban, N., y Taylor, V. (1993): «Mammography Utilization, Public Health Impact, and Cost-Effectiveness in the United States», en Annual Review of Public Health, 14, pp. 605-633.

\begin{abstract}
The aim of this article is to analyse the possible implication of variables in the health beliefs model and socio-demographic variables in participation in a breast cancer screening programme, which was carried out in Navarre within the framework of the European Pilot Network of Programmes for Early Detection of Breast Cancer, targeted at women between the ages of 45 and 65. A total sample of 708 women with normal health was used; 512 of them participated in the programme carried out in Navarre for early detection of breast cancer, while the remaining 196 did not take part in it. All the women were asked to complete a questionnaire on an individual basis.
\end{abstract}




\section{TEXTO CLÁSICO}

